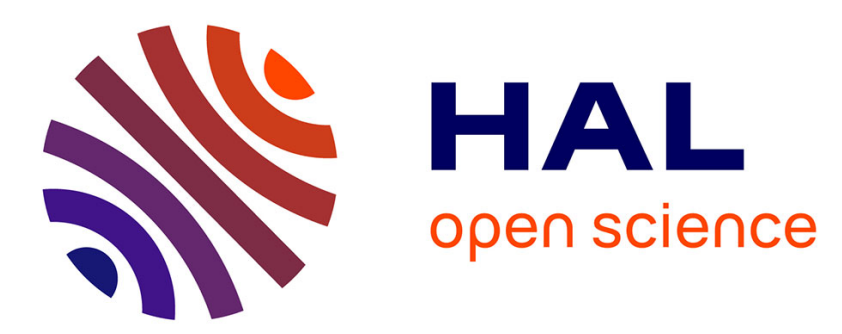

\title{
Comparison of Automatic Seed Generation Methods for Breast Tumor Detection Using Region Growing Technique
}

Ahlem Melouah

\section{- To cite this version:}

Ahlem Melouah. Comparison of Automatic Seed Generation Methods for Breast Tumor Detection Using Region Growing Technique. 5th International Conference on Computer Science and Its Applications (CIIA), May 2015, Saida, Algeria. pp.119-128, 10.1007/978-3-319-19578-0_10 . hal-01789934

\section{HAL Id: hal-01789934 \\ https://hal.inria.fr/hal-01789934}

Submitted on 11 May 2018

HAL is a multi-disciplinary open access archive for the deposit and dissemination of scientific research documents, whether they are published or not. The documents may come from teaching and research institutions in France or abroad, or from public or private research centers.
L'archive ouverte pluridisciplinaire HAL, est destinée au dépôt et à la diffusion de documents scientifiques de niveau recherche, publiés ou non, émanant des établissements d'enseignement et de recherche français ou étrangers, des laboratoires publics ou privés. 


\title{
Comparison of automatic seed generation methods for breast tumor detection using region growing technique
}

\author{
Ahlem Melouah \\ Department of Informatics, Labo LRI, \\ Badji-Mokhtar Annaba University \\ P.O.Box 12, 23000, Annaba, Algeria \\ ahlem.melouah@univ-annaba.dz
}

\begin{abstract}
Seeded Region Growing algorithm is observed to be successfully implemented as a segmentation technique of medical images. This algorithm starts by selecting a seed point and, growing seed area through the exploitation of the fact that pixels which are close to each other have similar features. To improve the accuracy and effectiveness of region growing segmentation, some works tend to automate seed selection step. In this paper, we present a comparative study of two automatic seed selection methods for breast tumor detection using seeded region growing segmentation. The first method is based on thresholding technique and the second method is based on features similarity. Each method is applied on two modalities of breast digital images. Our results show that seed selection method based on thresholding technique is better than seed selection method based on features similarity.
\end{abstract}

Keywords: medical image segmentation; medical informatics; automatic seed selection; region growing; tumor detection.

\section{INTRODUCTION}

The basic segmentation aim is to divide an image into different regions based on certain criteria. The regions with connected pixels of similar values can provide important cues for extracting semantic objects. Since, image segmentation is mainly used to locate an objects or an object boundary in an image thus it can be used in applications which involve a particular kind of object recognition such as breast tumor.

Though researchers introduced several images segmentation methods but, most of these methods are not suitable for medical images. Image segmentation using seeded region growing (SRG) technique has increasingly become a popular method because of its ability to involve a high-level knowledge of anatomical structures in seed selection process [Jianping et al. 2005]. In most of the region growing algorithms, all the neighbors need to be evaluated for the region to be grown. The region growing starts with a seed pixel and repeatedly adds new pixels as long as the segmentation criterion is satisfied [Deboeverie et al. 2013]. 
One of the most important factors in region growing process is seed pixel selection. Seed pixel is often chosen close to the center of the region of interest (ROI). For example, if we are to segment a tumor from the background, it is always advisable to select the seed point for the tumor in the middle of the tumor [Najarian and Splinter 2012]. If seeds are not properly selected, the final segmentation results would be definitely incorrect [Massich et al. 2011]. Despite the existence of many automatic seed selection methods, SRG algorithm still suffers from the problems of automatic seed generation [Mehnert and Jackway1997; Jianping et al. 2001].

In this paper, two automatic seed point selection methods are compared. The first method based on thresholding technique is proposed by Al-Faris et al. [Al-Faris et al. 2014]. The second method based on features similarity is proposed by Yuvaria and Ragupathy [Yuvarai and Ragupathy 2013]. The same data and the same criteria have been used in this comparison.

The rest of the paper is organized as follows: Section 2 describes experimental automatic seed selection methods. Section 3 gives a view on experimentation. Section 4 presents some results with discussion. Section 5 draws our conclusion.

\section{Automatic seed selection methods}

For the region growing to be effectively achieved, the crucial part is the position of the seed pixel which must be selected from where the region growing may start [Mesanovic et al. 2013]. Up to now, some works use a semi-automatic region growing algorithm and still need user interaction for seed selection. Other works are fully automatic and the user has only a verification role. Among these later works those proposed by Al-Faris et al. [Al-Faris et al. 2014] and Yuvarai et al. [Yuvarai and Ragupathy 2013]. Al-Faris et al. exposed an automatic seed selection method based on the thresholding technique. Yuvaria et al. developed an automatic seed selection method based on features similarity. The description of these two methods is detailed in the following.

\subsection{Seed Selection method based on Features Similarity (SSFS)}

In order to detect a mass in a mammogram using SRG segmentation, Yuvarai and Ragupathy proposed a new seed point selection method based on features similarity. Statistical features like mean, dissimilarity, sum average, sum variance and auto correlation are considered as significant features able to identify a mass. These features are computed and fixed for masses which have been previously identified by an expert. Seed selection process starts by initializing a mask, and then calculates its features from the regions within the mask. If the mask features do not match with the mass predefined features, the mask is therefore shift. Otherwise, the initial pixel of the mask is taken as seed point. 


\subsection{Seed Selection method based on Thresholding Technique (SSTT)}

Al-Faris et al. [Al-Faris et al. 2014] used SRG for breast MRI tumor segmentation with seed point selection based on the thresholding technique. A new algorithm is developed for automatic evaluation of the suitable threshold value. This algorithm searches for the maximum value in each row in the image and saves it temporarily. This process is repeated for all the rows until the last. Then, a summation of the temporarily stored values is calculated. The mean maximum raw is then calculated by dividing the summation value by the number of rows in the image. The resultant mean value will be considered as the threshold value for the binarization process. In order to remove the unwanted small white speckles in the image which do not belong to the ROI and enhance the boundary of the suspected regions, the morphological open operation (erosion followed by dilation operations) has been applied. To extract ROI, all the regions are ranked in an ascending order according to their density values. After, the highest region will be chosen as the main suspected region. The seed is the pixel of this main suspected region with maximum intensity value.

\section{Experiments}

\subsection{Dataset}

In this study, two databases with different modalities of breast digital images are considered:

1. RIDER breast MRI dataset downloaded from the National Biomedical Imaging Archive [10]. The dataset includes more than 1000 breast MRI images for five patients. All the images are axial 288 X 288 pixels. The dataset also includes Ground Truth (GT) segmentation which has been manually identified by a radiologist.

2. MiniMIAS database provided by the Mammographic Image Analysis Society (MIAS) [11]. MiniMIAS consists of a variety of normal mammograms as well as mammograms with different characteristics and several abnormalities. The mammograms are digitized at a resolution of $1024 \times 1024$ pixels and at 8-bit grey scale level. All the images include the locations of all the abnormalities that may be present.

\subsection{Seed point selection criterion}

Region growing is one of the most popular techniques for medical images segmentation due to its simplicity and good performance [Saad et al. 2012]. But, this performance is deeply influenced by seed point position. Therefore, selecting a good set of initial seeds is very important. To determine the good seed position, Massich [Massich et al. 2011] tested 10 areas-of-interest selected at different distances and orientations from the lesion center. The 10 tested areas are: The area 1 is the zone located outside the lesion; the areas from 2 to 5 are the zones situated on the boundaries of the lesion; the areas from 6 to 9 are the zones placed near the lesion center and, the area 10 is the lesion center. The best segmentation results are obtained by using the seed 
points located in area 10 . The segmentation performance decrease when the seed position moves away from the lesion center. Consequently, a seed point can be placed in three different areas:

1. Inside the ROI; in this situation, segmentation result is more and more accurate if seed position approximates the ROI center.

2. On the border of the ROI; in this situation, there are two possibilities, either the segmentation fails or success.

3. Outside the ROI; in this situation, the segmentation fails.

Figure 1 gives an example of these three situations. If the seed is centered in the ROI (figure 1.a), therefore the SRG segmentation well extracts the lesion (figure 1.e). If the seed is placed on the border of the ROI (Figure 1.b and Figure 1.c), therefore the SRG segmentation can success (figure 1.f) or can fail (figure 1.g). The SRG segmentation fails (figure 1.h) if the seed is placed outside the ROI (figure 1.d).

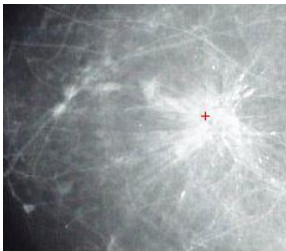

(a)

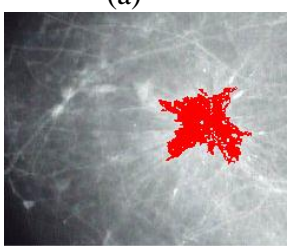

(e)

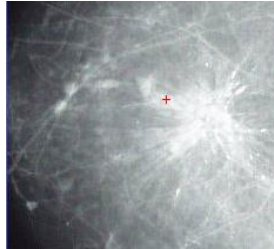

(b)

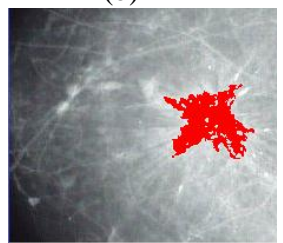

(f)

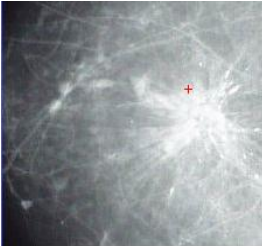

(c)

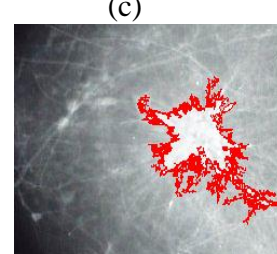

(g)

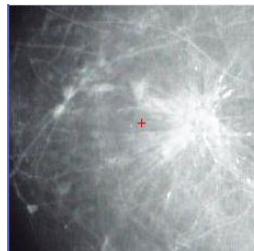

(d)

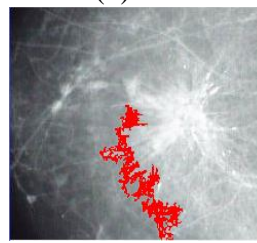

(h)

Fig. 1. Examples of different seed placement (left column) and correspondent segmentation results (right column)

To sum it up, the seed position can be considered as a good criterion in the comparison between automatic seed generation methods. The seed position is adequate if and only if the seed is placed inside the ROI. In addition, the best method is the method which generates seeds close the lesion center.

\section{$4 \quad$ Results and discussion}

Considering the fact that the initial seed selection has a great influence on the final segmentation accuracy, we propose a comparative study of two automatic seed selection methods: SSFS and SSTT. The behavior of the two methods was examined using a randomly selected dataset from MiniMIAS database and Rider database. We notify 
that, in region growing segmentation process, the same similarity measure and the same threshold value have been used for the two methods.

\subsection{Mammograms dataset}

To evaluate the performance of the experimental methods, 28 mammograms with tumors are taken from MiniMIAS database. The two methods are applied on each tested image.

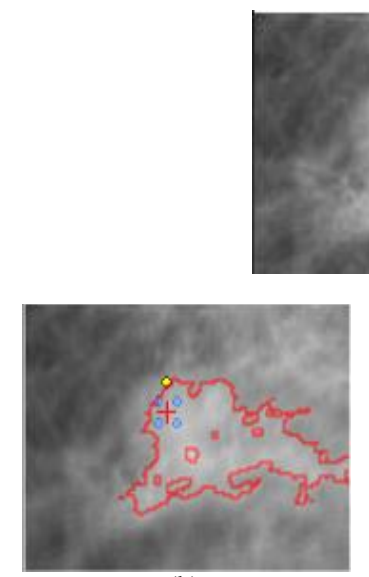

(b)

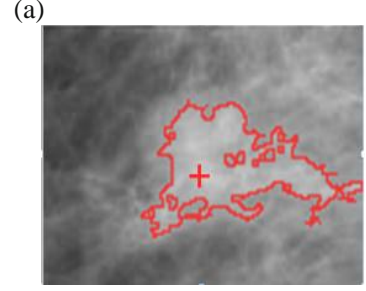

(c)

Fig. 2. Seed point generation example on mammogram. (a) Original image. (b) Seed generation result and segmentation result using SSFS. (c) Seed generation result and segmentation result using SSTT.

Figure 2 presents the results of the experimental methods on a mammogram test example. The original image is illustrated in Figure 2(a). Figure 2(b) shows seed generation result and segmentation result using SSFS method. Figure 2(c) shows seed generation result and segmentation result using SSTT method. We can see clearly that the two methods place correctly the seed inside the ROI, but at different positions.

According to the obtained results from all the tested mammograms, the seeds repartition area of each method is surrounded in figure 3. On a prototype image we have delimited separately the zones covered by SSFS and SSTT methods. The blue line delimits the SSFS zone and the red line delimits the SSTT zone. From this illustration three observations can be made:

1. The two methods SSFS and SSTT succeed in placing some seeds inside and close to the center of the ROI.

2. The SSTT method fails in some cases because it places a number of seeds outside the ROI.

3. The SSFS method gives better results than the SSTT method because in the worst case, the seed point is placed on the ROI boundary. 


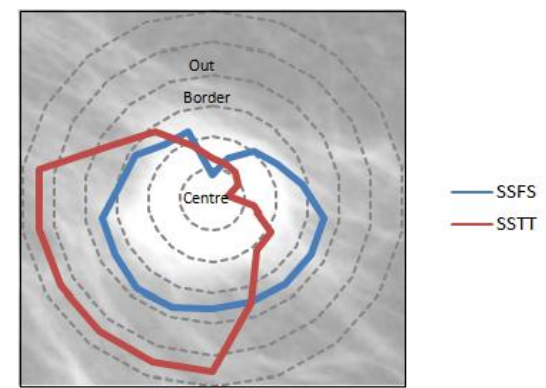

Fig. 3. Seeds repartition areas illustration for mammograms dataset

A priori, we can suppose that the SSFS method is more powerful than the SSTT method. But, when we look to the plot of the figure 4, this supposition becomes weak. The plot shows that the SSTT method places most seeds inside the ROI while the SSFS method places the majority of seeds on the ROI boundary.

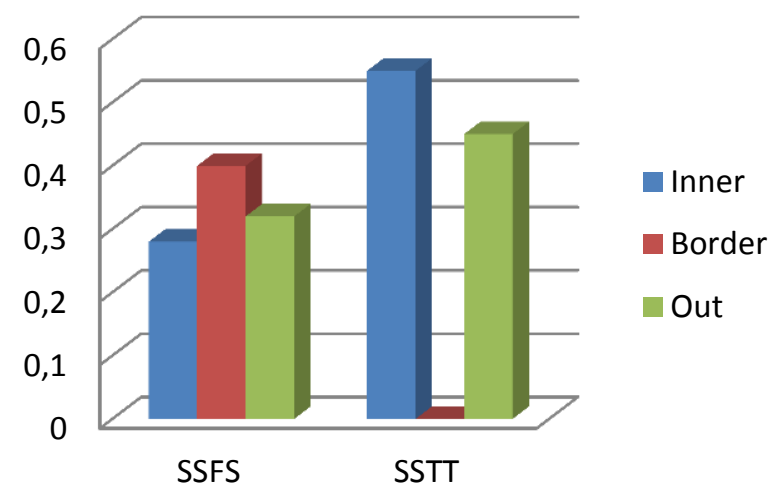

Fig. 4. Static results of the SSFS method and the SSTT method on the mammograms dataset.

From the results above, we conclude that the SSFS method can easily find the ROI but, has some difficulties to point their centre. On the contrary, the SSTT method is more powerful in locating the centre area if it success in detecting the ROI.

\subsection{Rider dataset}

To evaluate the performance of the experimental methods on another dataset, 20 breast IRM images with tumors were taken from Rider database. Seed point generation example by the two considered methods is shown in the following: 


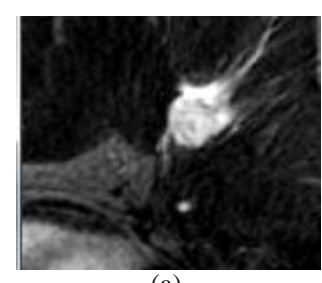

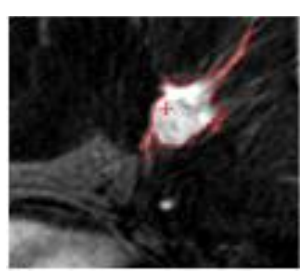

(b)

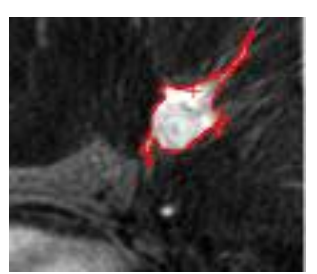

(c)

Fig. 5. Seed point generation example on breast IRM image (a) Original image (b) Seed generation result and segmentation result using SSFS. (c) Seed generation result and segmentation result using SSTT.

Figure 5 illustrates seed generation results and segmentation results using the two methods (SSFS and SSTT) on breast IRM example. The original image is illustrated in Figure 5(a). Figure 5(b) shows seed generation result and segmentation result using the SSFS method. Figure 5(c) exhibits seed generation result and segmentation result using the SSTT method. This example shows that the SSTT method places its seed farther from the centre of ROI than the SSFS method. This fact is not correct for all the seeds generated by the SSTT method. As it is presented in figure 6, the SSTT method places all its seeds in an area (represented by red line) included in the repartition area (represented by blue line) of the SSFS method. So, the SSFS method gives better results than the SSFS method in most cases.

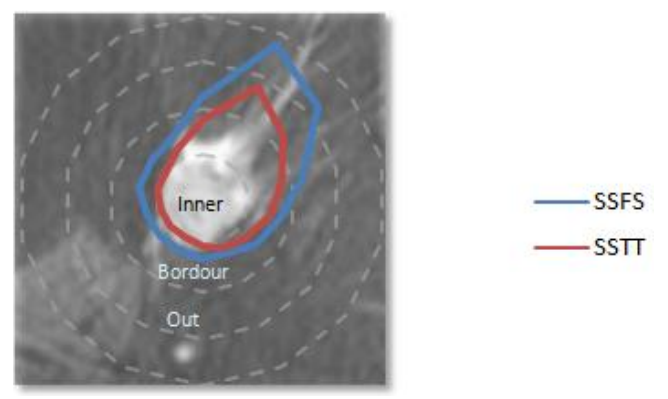

Fig. 6. Seeds repartition areas illustration for IRM dataset

The statistical data presented by the plot of the figure 7 confirms the efficiently of SSTT method in comparison with the SSFS method. 


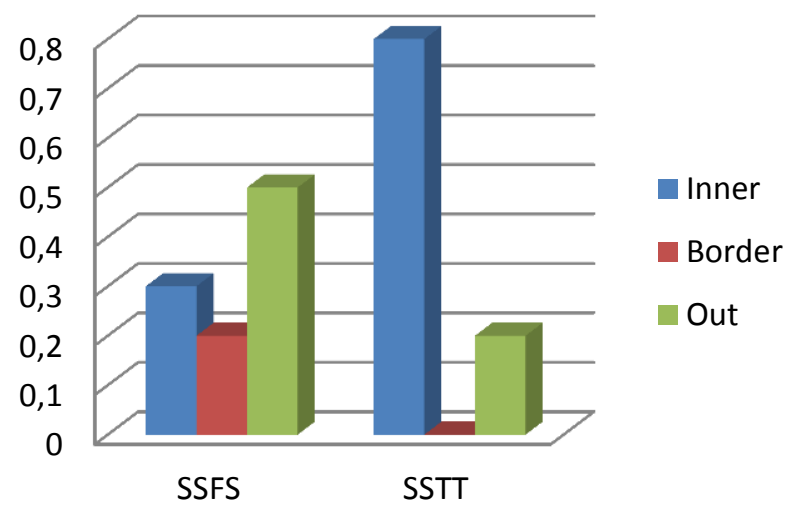

Fig. 7. Static results of the SSFS method and the SSTT method on the breast IRM dataset.

\subsection{Discussion}

In this work, two automatic seed selection methods have been studied and evaluated. The SSFS method and the SSTT method are tested using mammograms and breast IRM images. From the obtained results some conclusions can be drawn:

1. It is possible to apply the SSFS method and the SSTT method for both modalities. The SSTT method introduced for the IRM breast images gives good results for the mammograms as well. Despite the SSFS method had been developed, originally, for mammograms it gave also acceptable results for IRM breast images.

2. The SSTT method performs well if there are no undesirable regions. Undesirable regions are the regions with high intensity like labels, artifacts ...etc. So, if these undesirable regions are removed by using pre-processing stage, the performance of the SSTT method will certainly increase.

3. Masses predefined features values were carefully studied by the authors of the SSFS method. These references values which allow good tumors detection in mammograms can be inappropriate for IRM images. Hence, references values must be modified for each new used database this repeated modification will be an obstacle for the SSFS method adaptability. However, if references values are carefully selected, the SSFS performance will consequently augment. Unfortunately, it is very hard to fix the best references values for each used database.

4. The SSTT method has proved to be more efficient in matter of spotting the ROI centre compared to the SSFS method. The SSTT method selects the high intensity pixel as a seed, while the SSFS method selects the first pixel of the mask as a seed. The SSTT method seed selection criterion makes it possible to place the seed close to the ROI centre. On the other hand, the SSFS method seed selection criterion favours the seed placement on the ROI boundary. 


\section{Conclusion}

Since region growing technique often gives good segmentation results that correspond well to the observed edges, it is widely used in medical images. Typically, a seeded region growing algorithm includes two major steps. The first step is seed point generation by selecting an initial seed point somewhere inside the suspected lesion. The second step is region formation which starts from the seed point and grows progressively to fill a coherent region. As, region growing results are sensitive to the initial seeds, the accurate seed selection is very important for image segmentation. In this work, we have implemented, tested and evaluated two automatic seed selection methods. The SSTT method proposed by Al-Faris et al. is based on the thresholding technique. The SSFS method proposed by Yuvaria et al. is based on features similarity. The tests were elaborated on two different kinds of breast images modalities: mammograms and IRM. Both the SSTT and the SSFS methods deal well with mammograms. But, as far as IRM is concerned, the SSTT method performs better than SSFS method.

\section{References}

1. Jianping, F., Guihua, Z., Body, M., Hacid M.S.: Seeded region growing: an extensive and comparative study. Pattern Recognition Letters. 26(8), 1139-1156 ( 2005)

2. Deboeverie, F., Veelaert, P., Philips, W.: Image segmentation with adaptive region growing based on a polynomial surface model. Journal of Electronic Imaging,. 22(4), 1-13 (2013)

3. Najarian K., Splinter, R.: Biomedical signal and image processing. Second edition. CRC Press, Taylor \& Francis Group, United States of America (2012)

4. Massich, J., Meriaudeau, F., Pérez, E., Marti, R., Oliver, A., Marti, J.: Seed selection criteria for breast lesion segmentation in Ultra-Sound images. In Workshop on Breast Image Analysis In conjunction with MICCAI, 57-64 (2011)

5. Mehnert, A., Jackway, P.: An improved seeded region growing algorithm. Pattern Recognition letters. 18(10), 1065-1071 (1997)

6. Jianping, F., Yau, D.K.Y., Elmagarmid, A.K., Aref, W.G.: Automatic image segmentation by integrating color-based extraction and seeded region growing. IEEE Trans. Image Process. 10 (10), 1454-1466 (2001)

7. Al-Faris, A.Q., Umi Kalthum, N., MatIsa, N.A., Shuaib, I.L.: Computer-Aided Segmentation System for Breast MRI Tumour using Modified Automatic Seeded Region Growing, (BMRI-MASRG). J Digit Imaging, 1 27, 133-144 (2014)

8. Yuvarai, K., Ragupathy, U.S.: Automatic Mammographic Mass Segmentation based on Region Growing Technique. In: 3rd International Conference on Electronics, Biomedical Engineering and its Applications (ICEBEA'2013), pp. 29-30. Apri,1 Singapore (2013)

9. Mesanovic, N., Huseinagic, H., Kamenjakovic, S.: Automatic Region Based Segmentation and Analysis of Lung Volumes from CT Images. International Journal of Computer Science And Technology. 4(2), 48-51, June (2013)

10. US National Cancer Institute: reference image database to evaluate therapy response (RIDER) MRI breast, 2007 The Cancer Imaging Archive (TCIA), Avaible from http://cancerimagingarchive.net./about-archive.html 
11. http://peipa.essex.ac.uk/info/mias.html

12. Mohd Saad, N., Abu-Bakar, S.A.R., Muda, S., Mokji, M., Abdullah. A.R..: Automated Region Growing for Segmentation of Brain Lesion in Diffusion-weighted MRI, In. Proceeding of the international MultiConference of Enginneers and Computer Scientists, vol. 1, pp. 14-16. March, Hong Kong (2012) 